\title{
Properties of mesoscopic superconducting thin-film rings. London approach
}

\author{
V. G. Kogan, John R. Clem \\ Ames Laboratory - DOE and Department of Physics and Astronomy, Iowa State University, Ames Iowa 50011 \\ R. G. Mints \\ School of Physics and Astronomy, Raymond and Beverly Sackler \\ Faculty of Exact Sciences, Tel Aviv University, Tel Aviv 69978, Israel
}

(Dated: May 3, 2022)

\begin{abstract}
Superconducting thin-film rings smaller than the film penetration depth (the Pearl length) are considered. The current distribution, magnetic moment, and thermodynamic potential $\mathcal{F}(H, N, v)$ for a flat, washer-shaped annular ring in a uniform applied field $H$ perpendicular to the film are solved analytically within the London approach for a state with winding number $N$ and a vortex at radius $v$ between the inner and outer radii.
\end{abstract}

PACS numbers: 74.78.-w,74.78.Na

\section{INTRODUCTION}

Small flat rings made of thin superconducting films are of interest for a variety of mesoscopic experiments .1 .2 SQUID-type devices is an example; another one is the study of fluxoid dynamics in rings, as discussed by Kirtley et al. $\frac{3}{2}$ The basic physics of such rings is governed by flux quantization, as in the Little-Parks experiment; see, e.g., Ref. 4 or later publications ${ }^{5,6.7 .8}$ based on the GinzburgLandau theory.

There is a resurgence of experimental interest in this subject: The interacting dipole moments in an array of superconducting rings provide a model system for magnetism in Ising antiferromagnets $\frac{1.2}{}$ Moreover, there is considerable interest in quantum coherence effects in superconducting rings and their arrays for potential applications in quantum computing $\underline{9,10.11}$

Many quantitative details specific to the thin-film geometry can be treated within the London approach, which is not bound by the strict temperature restriction of Ginzburg-Landau models. In the thin-film limit, for which the London penetration depth $\lambda$ obeys $\lambda \gg d$, the film thickness, the fields and currents are governed by the Pearl length $\Lambda=2 \lambda^{2} / d{ }^{12}$ As we show below, when the inner and outer radii $a$ and $b$ of the annular ring are smaller than $\Lambda$, it is possible to obtain analytic solutions for the energy of the ring in a uniform magnetic field $H$ applied perpendicular to the ring plane with a vortex sitting in an arbitrary position at the annular region between $a$ and $b$. The motion of vortices between $a$ and $b$ provides the means for the ring to switch between discrete states with different winding numbers $N$ defined below. Study of these transitions is relevant for understanding the telegraph noise observed in multiply connected mesoscopic superconducting devices in general and in thin-film rings in particular ${ }^{\underline{3}}$

\section{CURRENT DISTRIBUTION}

Let us consider a small thin-film ring of thickness $d \ll$ $\lambda$ situated in the plane $z=0$ with inner and outer radii $a$ and $b$, where $b$ is much smaller than the Pearl length $\Lambda=2 \lambda^{2} / d$. The ring is in a uniform applied field $H$ perpendicular to the film plane; it may contain a vortex (or antivortex), the position of which can be taken as $x=v, y=0$ (with the origin at the ring center and $a<v<b)$. The London equations for the local magnetic field $\boldsymbol{b}$ in the film interior read ${ }^{13}$

$$
\boldsymbol{b}+\frac{4 \pi \lambda^{2}}{c} \operatorname{curl} \boldsymbol{j}= \pm \phi_{0} \hat{\boldsymbol{z}} \delta(x-v, y)
$$

Here, $\boldsymbol{j}$ is the current density, $\hat{\boldsymbol{z}}$ is the unit vector perpendicular to the film plane, and $\phi_{0}=\pi \hbar c /|e|$ is the value of the flux quantum. The upper sign holds for a vortex whereas the lower one is for an antivortex, the convention retained throughout this paper. Averaging this over the thickness $d$, one obtains

$$
b_{z}+\frac{2 \pi \Lambda}{c} \operatorname{curl}_{z} \boldsymbol{g}= \pm \phi_{0} \delta(\boldsymbol{r}-\boldsymbol{v})
$$

where $\boldsymbol{g}(\boldsymbol{r})$ is the sheet current density, $\boldsymbol{r}=(x, y)$, and $\boldsymbol{v}=(v, 0)$. Equations (11) and (2) are valid everywhere in the film except within a distance of the order of the coherence length $\xi$ (vortex core) from the vortex or antivortex axis, where the London equation (11) no longer holds.

The distribution $\boldsymbol{g}(\boldsymbol{r})$ can be found by solving Eq. (2), combined with the continuity equation and the BiotSavart integral that relates the field $b_{z}$ to the surface current:

$$
\operatorname{div} \boldsymbol{g}=0, \quad b_{z}(\boldsymbol{r})=\int\left[\boldsymbol{g}\left(\boldsymbol{r}^{\prime}\right) \times \boldsymbol{R} / c R^{3}\right]_{z} d^{2} \boldsymbol{r}^{\prime}+H
$$

where $\boldsymbol{R}=\boldsymbol{r}-\boldsymbol{r}^{\prime}$. 


\section{A. The stream function}

In principle, Eqs. (2) and (3) determine the current distribution. To solve these equations for the general case is a difficult task, even for a disk ${ }^{14}$ However, for small samples, as in our case for which $b \ll \Lambda$, the problem can be solved ${ }^{15}$ To this end, let us introduce a scalar stream function $G(\boldsymbol{r})$, such that

$$
\boldsymbol{g}=\operatorname{curl}(G \hat{\boldsymbol{z}})
$$

The first of Eqs. (3) is then satisfied. It is easily seen that the contours $G(x, y)=$ const coincide with the current streamlines. Substituting Eq. (4) into (2), we obtain:

$$
\frac{2 \pi \Lambda}{c} \nabla^{2} G=\mp \phi_{0} \delta(\boldsymbol{r}-\boldsymbol{v})+b_{z}
$$

The radial component of the current at the ring edges must be zero; this means that the values of $G(x, y)$ at the edges are constants:

$$
G(r=a, \varphi)=G_{a}, \quad G(r=b, \varphi)=G_{b}
$$

where $(r, \varphi)$ are polar coordinates. Since $g_{\varphi}=-\partial G / \partial r$, the total counterclockwise current (in the $\varphi$ direction) around the ring is

$$
I=G_{a}-G_{b}
$$

The self-field of the ring currents within the ring can be estimated using the Biot-Savart law: $\int d^{2} \boldsymbol{r} g / c R^{2} \sim g / c$. Substitution of $b_{z} \sim H+g / c$ into Eq. (2) reveals that the self-field can be disregarded because $R \ll \Lambda$; i.e., we can set $b_{z}=H$ in Eq. (5):

$$
\frac{2 \pi \Lambda}{c} \nabla^{2} G=\mp \phi_{0} \delta(\boldsymbol{r}-\boldsymbol{v})+H \text {. }
$$

Since this equation is linear, we can look for a solution of the form $G=G_{v}+G_{H}$, such that $G_{v}$ satisfies

$$
\nabla^{2} G_{v}=\mp \frac{c \phi_{0}}{2 \pi \Lambda} \delta(\boldsymbol{r}-\boldsymbol{v})
$$

and

$$
\nabla^{2} G_{H}=\frac{c}{2 \pi \Lambda} H
$$

One can say that $G_{v}$ describes the currents due to the vortex or antivortex, whereas $G_{H}$ is the response to the applied field.

The boundary conditions (6) are imposed on the sum $G_{v}+G_{H}$. It is convenient to require that

$$
G_{v}(a)=G_{v}(b)=0,
$$

and

$$
G_{H}(a)=G_{a}, \quad G_{H}(b)=G_{b}
$$

In a uniform field $H, G_{H}$ can be taken as cylindrically symmetric. Aside from an unimportant additive constant,

$$
G_{H}(r)=\frac{c H}{8 \pi \Lambda} r^{2}+G_{0} \ln \frac{r}{a},
$$

where the constant $G_{0}$ is expressed in terms of the total current (7):

$$
I=-\frac{c H\left(b^{2}-a^{2}\right)}{8 \pi \Lambda}-G_{0} \ln \frac{b}{a} .
$$

To evaluate $I$ we use the London equation in the form

$$
\boldsymbol{g}=-\frac{c \phi_{0}}{4 \pi^{2} \Lambda}\left(\nabla \theta+\frac{2 \pi}{\phi_{0}} \boldsymbol{A}\right)
$$

Here $\theta$ is the order parameter phase, the topology of which plays a major role in our problem.

When the ring is traversed around a circle of radius $r$ in the positive direction of the azimuth $\varphi$, the phase changes by $-2 \pi N$, where $N$ is an integer which is commonly called the winding number or the vorticity. If there are no vortices in the annulus, the integer $N$ is the same for any contour within the annulus and we consider the state as having winding number $N$. In other words, the state of the system is characterized by the integer $N$ and the continuous variable $H$. In zero field, $N>0$ corresponds to positive currents $g_{\varphi}$ and positive magnetic moments $\mu_{z}$.

In the presence of a vortex, however, the situation is different. For contours encircling the ring's hole, the winding number is $N$ at contours that do not include the vortex position; the number is $N+1$ for those that do. The state is now characterized by the vortex position $\boldsymbol{v}$ in addition to $N$ and $H$. In this paper, when the state of the ring with a vortex is characterized by the variables $N, H$, and $v$, it is implied that the integer $N$ describes the phase topology on contours that do not include the vortex. The generalization to antivortices is obvious.

Coming back to evaluation of the total current, we integrate $I=\int_{a}^{b} g_{\varphi}(r, \varphi) d r$ over $\varphi$ to get:

$$
I=\int_{a}^{b} \bar{g}_{\varphi}(r) d r
$$

where the azimuthal average of $g_{\varphi}(r, \varphi)$ is

$$
\bar{g}_{\varphi}(r)=\int_{0}^{2 \pi} g_{\varphi} \frac{d \varphi}{2 \pi}=\frac{c \phi_{0}}{4 \pi^{2} \Lambda}\left(\frac{N}{r}-\frac{\pi H}{\phi_{0}} r\right),
$$

for $r<v$, and

$$
\bar{g}_{\varphi}(r>v)=\frac{c \phi_{0}}{4 \pi^{2} \Lambda}\left(\frac{N \pm 1}{r}-\frac{\pi H}{\phi_{0}} r\right) .
$$

We now readily evaluate the total current and the constant $G_{0}$ :

$$
G_{0}=-\frac{c \phi_{0}}{4 \pi^{2} \Lambda}\left[N \pm \frac{\ln (b / v)}{\ln (b / a)}\right]
$$


Note that $G_{0}$, which determines the part $G_{H}$ of the stream function, depends on whether or not a vortex is present at the ring and on its position. In other words, the current distribution is not a simple superposition of the currents generated by a vortex in zero field and of those existing in the state $(N, H)$ in the vortex absence.

\section{B. Electrostatic analogy and exact solution}

To find the solution of Eq. (9) for the vortex-generated stream function $G_{v}$ subject to the boundary conditions (11), we observe that the problem is equivalent to the two-dimensional one for the electrostatic potential generated by a line charge $\pm c \phi_{0} / 8 \pi^{2} \Lambda$ at the point $\boldsymbol{v}$ situated between two coaxial grounded metallic cylinders with radii $a$ and $b$. The necessary conformal mapping procedure is given in Ref. 16:

$$
\begin{aligned}
G_{v}(w, v) & = \pm \frac{c \phi_{0}}{4 \pi^{2} \Lambda} \operatorname{Re}\left[\ln \frac{A(w, v)}{B(v)}\right], \quad w=r e^{i \varphi} \\
A(w, v) & =\frac{\operatorname{cn}[2 \gamma \ln (v / a), m]}{\operatorname{sn}[2 \gamma \ln (v / a), m]}-\frac{\operatorname{cn}[\gamma \ln (v / w), m]}{\operatorname{sn}[\gamma \ln (v / w), m]} \\
B(v) & =\frac{\operatorname{cn}[2 \gamma \ln (v / a), m]}{\operatorname{sn}[2 \gamma \ln (v / a), m]}-\frac{\operatorname{cn}[\gamma \ln (v / a), m]}{\operatorname{sn}[\gamma \ln (v / a), m]}
\end{aligned}
$$

where $\operatorname{cn}(x), \operatorname{sn}(x)$ are the Jacobi elliptic functions, $\gamma=$ $\mathrm{K}(m) / \ln (b / a)$ with $\mathrm{K}(m)$ being the complete elliptic integral (in the notation of Ref. 17), and the parameter $m$ is chosen to satisfy

$$
\mathrm{K}(1-m) \ln (b / a)=\pi \mathrm{K}(m) .
$$

As an example, we find that for $b / a=2$ (such rings were studied in Ref. 3 ), $m=1.048 \times 10^{-5}$, and $\gamma=2.266$.

For rings with $1<b / a<2$, we have $0<\ln (b / a)<1$. Solving Eq. (21) numerically, one can see that $m \ll 1$. Expanding functions $\mathrm{K}(m)$ and $\mathrm{K}(1-m)$ for small $m$, one obtains with a high accuracy:

$$
m=16 \exp \left[-\frac{\pi^{2}}{\ln (b / a)}\right], \quad \gamma=\frac{\pi}{2 \ln (b / a)} .
$$

One can now set $m=0$ in Eq. (20) to obtain

$$
G_{v} \approx \pm \frac{c \phi_{0}}{4 \pi^{2} \Lambda} \operatorname{Re}\left\{\ln \frac{\sin \left[\pi \ln \left(v w / a^{2}\right) / 2 \ln (b / a)\right]}{\sin [\pi \ln (v / w) / \ln (b / a)]}\right\}
$$

At the vortex position $r=v, \quad \varphi=0$, this function diverges logarithmically. One can find $G_{v}(\boldsymbol{v})$ [and the self-energy $\left.\epsilon_{v}=\phi_{0}\left|G_{v}(v)\right| / 2 c\right]$ by introducing the standard cutoff at a distance $\xi$ from the vortex axis at $\boldsymbol{v}$ :

$$
\epsilon_{v} \approx \frac{\phi_{0}^{2}}{8 \pi^{2} \Lambda} \ln \left[\frac{2 v \ln (b / a)}{\pi \xi} \sin \frac{\pi \ln (v / a)}{\ln (b / a)}\right] .
$$

The relative difference between this expression and the exact energy is less than $2 \times 10^{-12}$ for $b / a \leq 2.18$ Formally,
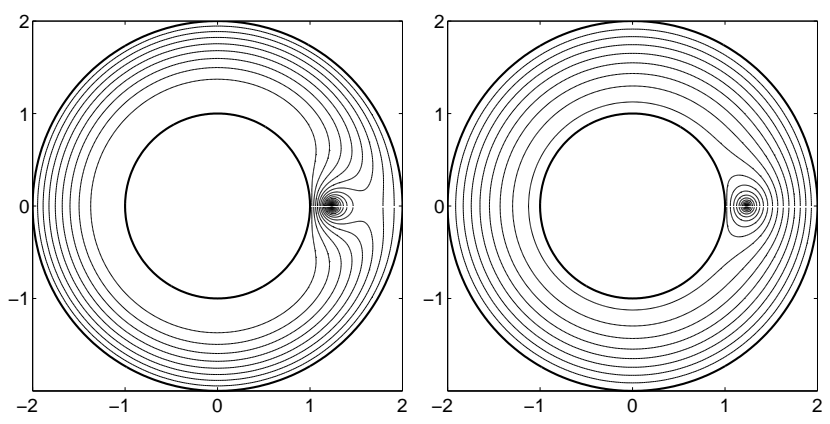

FIG. 1: Current streamlines in a ring with $b / a=2, N=2$, and vortex or antivortex position $v / a=1.2$ in the normalized field $h=H / H_{0}=2.3$ [see Eq. (39)]. The streamlines are plotted as contours of $G=G_{v}+G_{H}=$ const. The left panel is for a vortex; the streamlines shown that encircle the hole have clockwise flow, whereas the flow around the vortex is counterclockwise. The right panel shows an antivortex; all streamlines shown have clockwise flow.

the logarithmic factor in Eq. (24) goes to $-\infty$ if $v \rightarrow a$ or $v \rightarrow b$. However, this expression fails when the vortex is within roughly $\xi$ of the inner or outer radius. In fact, $\epsilon_{v}$ of Eq. (24) becomes equal to zero at $v_{a}=a(1+\xi / 2 a)$ and $v_{b}=b(1-\xi / 2 a)$.

Thus, the problem of the current distribution in the ring is solved: the vortex-generated part $G_{v}$ of the stream function is given in Eqs. (20), while $G_{H}$ is determined by Eqs. (13) and (19). The current streamlines are given by the contours $G(r, \varphi)=G_{v}(r, \varphi)+G_{H}(r)=$ const; Fig. 1 shows examples of current streamlines for a vortex and an antivortex.

It is worth mentioning that the same method based on application of conformal mapping to problems of the two-dimensional electrostatics can be utilized to obtain current distributions for $n$ vortices equally spaced along a circle of radius $a<v<b$ of the ring 16

\section{ENERGY}

\section{A. Zero applied field}

Let us first consider the energy $E(N, v)$ of the ring with a vortex at $v$ in the state $N$ in zero applied field. This energy is a sum of the magnetic and kinetic contributions. The magnetic part is 19

$$
\begin{aligned}
E_{m} & =\frac{1}{2 c} \int \boldsymbol{A} \cdot \boldsymbol{g} d^{2} \boldsymbol{r} \\
& =-\frac{1}{2 c} \int\left(\frac{2 \pi \Lambda}{c} \boldsymbol{g}+\frac{\phi_{0}}{2 \pi} \nabla \theta\right) \cdot \boldsymbol{g} d^{2} \boldsymbol{r}
\end{aligned}
$$

where we use Eq. (15) to express the vector potential $\boldsymbol{A}$ at the ring in terms of $\boldsymbol{g}$ and the phase $\theta$. The supercurrent kinetic energy $E_{k}$ is the integral over the film volume of the quantity $2 \pi \lambda_{L}^{2} j^{2} / c^{2}=\pi \Lambda g^{2} / c^{2} d{ }^{13}$ We find readily 
that this energy is equal in value and opposite in sign to the term containing $g^{2}$ in Eq. (25), so that

$$
E(N, v) \equiv E_{m}+E_{k}=-\frac{\phi_{0}}{4 \pi c} \int(\nabla \theta \cdot \boldsymbol{g}) d^{2} \boldsymbol{r} .
$$

The integrand here can be further transformed in terms of the stream function $G$ :

$$
\nabla \theta \cdot \operatorname{curl}(G \boldsymbol{z})=\operatorname{div}(G \boldsymbol{z} \times \nabla \theta)+G \boldsymbol{z} \cdot \operatorname{curl} \nabla \theta .
$$

Substituting this into Eq. (26), we use Gauss's theorem to evaluate the contribution of the first term:

$$
\begin{aligned}
& \int \operatorname{div}(G \boldsymbol{z} \times \nabla \theta) d^{2} \boldsymbol{r} \\
= & \int_{0}^{2 \pi}\left[-\left.G(b, \varphi) \frac{\partial \theta}{\partial \varphi}\right|_{b}+\left.G(a, \varphi) \frac{\partial \theta}{\partial \varphi}\right|_{a}\right] d \varphi \\
= & 2 \pi\left[(N \pm 1) G_{b}-N G_{a}\right] .
\end{aligned}
$$

Here we have used the boundary conditions (6) and the phase change is $-2 \pi N$ upon circling the inner radius and $-2 \pi(N \pm 1)$ the outer radius, provided a single vortex (antivortex) is present in the annulus.

Integrating the second term of Eq. (27), we use the basic quantization property of the phase $\left[\operatorname{curl}_{z} \nabla \theta=\right.$ $\mp 2 \pi \delta(\boldsymbol{r}-\boldsymbol{v})]$, take the curl of Eq.(15), and compare the result with Eq. (2). Then, we obtain the magnetic and kinetic energy of the persistent currents in the ring:

$$
E(N, v)=\frac{\phi_{0}}{2 c}\left[G(v)-(N \pm 1) G_{b}+N G_{a}\right]
$$

Note that $G(v)=G_{v}(v)+G_{H}(v)$, and the vortex selfenergy is $\epsilon_{v}=\phi_{0}\left|G_{v}(v)\right| / 2 \pi$ (see Ref. 15). Utilizing Eqs. (13) and (19), we obtain:

$$
E(N, v)=\epsilon_{v}(v)+\epsilon_{0}\left[N \pm \frac{\ln (b / v)}{\ln (b / a)}\right]^{2}
$$

where we introduce the energy scale

$$
\epsilon_{0}=\frac{\phi_{0}^{2} \ln (b / a)}{8 \pi^{2} \Lambda}
$$

If $v=b$, i.e., if there is no vortex in the annulus, we have

$$
E_{0}=\frac{\phi_{0}}{2 c}\left(G_{a}-G_{b}\right) N=\frac{\phi_{0}}{2 c} N I
$$

\section{B. Magnetic moment}

By definition, the $z$ directed magnetic moment reads: ${ }^{19}$

$$
\mu=\frac{1}{2 c} \int_{a}^{b} d r r^{2} \int_{0}^{2 \pi} d \varphi g_{\varphi}(r, \varphi)=\frac{\pi}{c} \int_{a}^{b} r^{2} \bar{g}_{\varphi}(r) d r
$$

This is easily evaluated using Eqs. (17), (18):

$$
\mu=\frac{\phi_{0}\left(b^{2}-a^{2}\right)}{8 \pi \Lambda}\left(N \pm \frac{b^{2}-v^{2}}{b^{2}-a^{2}}\right)-\frac{H}{16 \Lambda}\left(b^{4}-a^{4}\right) .
$$

It is worth noting that the magnetic moment is evaluated here without need to employ the explicit current distribution which could have been obtained from the known stream function $G_{v}+G_{H}$.

Setting $v=b$, we obtain the moment $\mu_{0}$ in the "pure" state $N$ with no vortex at the ring:

$$
\mu_{0}(N, H)=\frac{\phi_{0}\left(b^{2}-a^{2}\right)}{8 \pi \Lambda} N-\frac{H}{16 \Lambda}\left(b^{4}-a^{4}\right) .
$$

The result can be verified by direct calculation of $\mu$ using the current distribution in the absence of vortices. If we set $v=a$ in (34), we obtain $\mu_{0}(N \pm 1, H)$.

It is instructive to observe that the exact moment (34) in the presence of a vortex at $v$ can be considered as a sum of the magnetic moment of a vortex-free ring with inner and outer radii $a$ and $v$ having winding number $N$ and another ring with inner and outer radii $v$ and $b$ with vorticity $N+1$.

\section{Free energy in field}

The thermodynamic potential $\mathcal{F}$ which is minimum in equilibrium at a given applied field is defined by a differential relation

$$
\delta \mathcal{F}=-\boldsymbol{\mu} \cdot \delta \boldsymbol{H}=-\mu \delta H,
$$

where $\boldsymbol{\mu}(N, v, H)$ is the system magnetic moment 19 Using Eq. (34), we readily obtain $\mathcal{F}$ by integrating Eq. (36) over $H$ from 0 to $H$ :

$$
\begin{aligned}
\mathcal{F}_{ \pm}=E(N, v) & -\frac{\phi_{0} H\left(b^{2}-a^{2}\right)}{8 \pi \Lambda}\left(N \pm \frac{b^{2}-v^{2}}{b^{2}-a^{2}}\right) \\
& +\frac{H^{2}}{32 \Lambda}\left(b^{4}-a^{4}\right),
\end{aligned}
$$

where the zero-field energy $E(N, v)$ is given in Eq. (30). After simple algebra we arrive at our main result,

$$
\begin{aligned}
\mathcal{F}_{ \pm}(N, v, H) & =\epsilon_{v}(v)+\epsilon_{0}\left[\left(N \pm \frac{\ln (b / v)}{\ln (b / a)}\right)^{2}\right. \\
& \left.-2 h\left(N \pm \frac{b^{2}-v^{2}}{b^{2}-a^{2}}\right)+h^{2} \chi\right]
\end{aligned}
$$

where we have introduced a dimensionless field $h=$ $H / H_{0}$, where

$$
H_{0}=\frac{2 \phi_{0} \ln (b / a)}{\pi\left(b^{2}-a^{2}\right)}
$$

and a geometric factor $\chi$ given by

$$
\chi=\frac{b^{2} / a^{2}+1}{b^{2} / a^{2}-1} \ln \frac{b}{a} .
$$


For a narrow ring $b / a-1=\eta \ll 1, \chi=1+\mathcal{O}\left(\eta^{2}\right)$. With increasing $b / a, \chi$ grows slowly: for $b / a=2, \chi \approx 1.155$, and $\chi \approx \ln (b / a)$ for large values of $b / a$.

\section{Vortex-free state}

For the vortex-free state, Eq. (38) yields:

$$
\mathcal{F}_{0}(N, h)=\epsilon_{0}\left(N^{2}-2 N h+h^{2} \chi\right) .
$$

It is seen that $\mathcal{F}_{0}\left(N_{1}, h\right)=\mathcal{F}_{0}\left(N_{2}, h\right)$ at the field $h=$ $\left(N_{1}+N_{2}\right) / 2$ for any $N_{1}$ and $N_{2}$. In particular, for $N_{1}=N$ and $N_{2}=N+1$, the energies $\mathcal{F}_{0}(N, h)=\mathcal{F}_{0}(N+1, h)$ at $h=N+1 / 2$. In other words, at this field the system might be in either of the states $N$ or $N+1$ having the same energy.

One can readily check that the thermodynamic potential $\mathcal{F}_{0}(N, h)$ of the vortex-free state coincides with the kinetic energy of the supercurrents in the ring.

A transition from a state with vorticity $N$ to one with vorticity $N+1$ can happen when a vortex, carrying unit vorticity, enters at the outer radius, crosses the ring, and annihilates at the inner radius. Alternatively, such a transition can be accomplished, starting with initial vorticity $N$, when an antivortex, carrying vorticity -1 , enters the annulus at the inner radius $a$, crosses the ring, and annihilates at the outer radius $b$, leaving behind vorticity $N+1$ trapped in the ring.

The field dependence of $\mathcal{F}_{0}$ for $0 \leq N \leq 4$ is shown in Fig. 2 which illustrates the above features. It shows that in fields $N-1 / 2<h<N+1 / 2$, the minimum energy belongs to the state $N$. However, the first excited state is $N-1$ for $N-1 / 2<h<N$, whereas it is $N+1$ for $N<h<N+1 / 2$. It should be also noted that in a given field $h$, the ground state winding number is the integer nearest to $h$.

Although the ground state vorticity $N$ changes with field, the energy difference between the lowest and the "first excited state" is, in fact, periodic in $h$ with the period $\Delta h=1$. It is easy to check that the difference $\mathcal{F}_{0}(N, h)-\mathcal{F}_{0}(N-1, h)=\mathcal{F}_{0}(N+1, h+1)-\mathcal{F}_{0}(N, h+1)$. This fact has implications for the transition probabilities from the ground state to the nearest excited state.

It is worth observing that the energy (38) differs from that of the vortex-free state, $\mathcal{F}_{0}$ of Eq. (41), by the vortex self-energy $\epsilon_{v}(v)$ and by the addition to $N$ of the $v$-dependent terms that vary from unity to zero as the vortex moves from $a$ to $b$.

\section{E. Potential barriers for vortices crossing the ring}

We are now in a position to evaluate the energy barrier that must be overcome for a vortex or an antivortex to move between the inner and outer radii of the ring. Since $G_{v}=0$ at both $a$ and $b$, we have at $v=b$ :

$$
\mathcal{F}_{+}(N, b)=\mathcal{F}_{-}(N, b)=\mathcal{F}_{0}(N),
$$

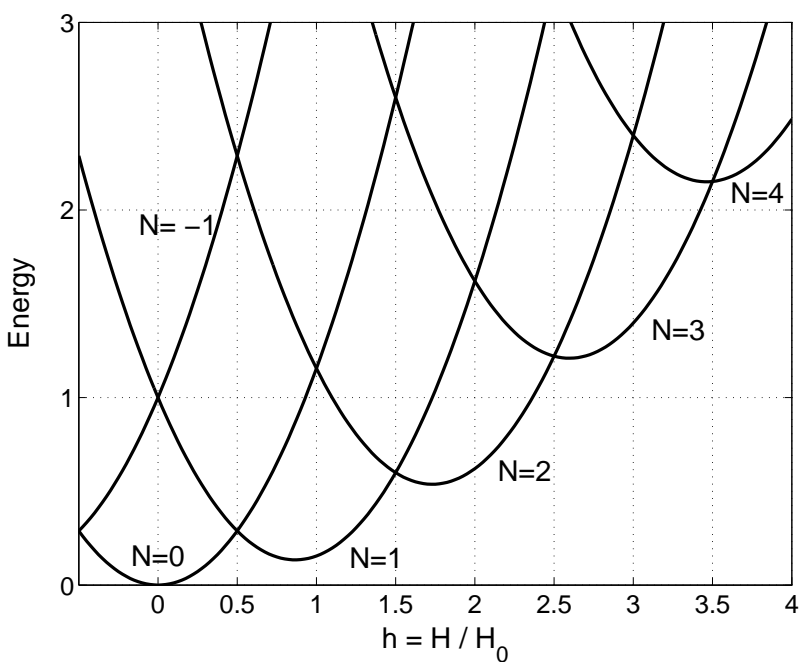

FIG. 2: The energy $\mathcal{F}_{0}$ of vortex-free states of a ring with $b / a=2$ for vorticities $N$ from 0 to 4 versus applied field. The energy and field units are defined in Eqs. (31) and (39). Note that e.g. for $0.5<h<1.5, N=1$ corresponds to the ground state. The nearest "excited" state is $N=0$ for $0.5<h<1$, whereas for $1<h<1.5$ it is $N=2$.

where the energy $\mathcal{F}_{0}(N)$ of the vortex-free states is given by Eq. (41); the subscript "+" is for a vortex, whereas "-" stands for an antivortex (the field argument $H$ is suppressed for brevity). In other words, the addition of either a vortex or an antivortex at the outer radius does not change the vorticity of the system. On the other hand, at $v=a$ we have:

$$
\mathcal{F}_{+}(N, a)=\mathcal{F}_{0}(N+1), \quad \mathcal{F}_{-}(N, a)=\mathcal{F}_{0}(N-1) ;
$$

i.e., moving a vortex (antivortex) from $b$ to $a$ changes the vorticity by $+1(-1)$, an obvious consequence of the system topology.

Starting from a vortex-free state $N$, the motion of a vortex between $a$ and $b$ can change the system energy in two ways. If a vortex moves in, starting from the outer radius $b$, the spatial dependence of the potential energy barrier against vortex entry is given by the difference

$$
\mathcal{F}_{+}(N, v)-\mathcal{F}_{0}(N)=V_{\text {in }}^{+}(N, v),
$$

so that $V_{i n}^{+}(N, b)=0$, while $V_{i n}^{+}(N, a)=\epsilon_{0}(2 N+1-2 h)$, which corresponds to the energy of transition between "pure" states $N$ and $N+1$. On the other hand, if a vortex moves out, starting from the inner radius $a$, the spatial dependence of the potential energy barrier against vortex exit is given by the function

$$
V_{\text {out }}^{+}(N, v)=\mathcal{F}_{+}(N-1, v)-\mathcal{F}_{0}(N),
$$

such that $V_{\text {out }}^{+}(N, a)=0$, while $V_{\text {out }}^{+}(N, b)=\epsilon_{0}(2 h-$ $2 N+1$ ), which corresponds to the energy of transition from the state $N$ to the state $N-1$.

When $h=N+1 / 2$, we obtain $V_{i n}^{+}(N, a)=V_{i n}^{+}(N, b)=$ $0, V_{\text {out }}^{+}(N+1, b)=V_{\text {out }}^{+}(N+1, a)=0$ for any $N$. More- 
over, the potential barriers $V_{i n}^{+}(N, v)$ and $V_{o u t}^{+}(N+1, v)$ are identical.

Similarly, the motion of an antivortex between $a$ and $b$ can change the system energy in two ways. If an antivortex moves in, starting from the outer radius $b$, the spatial dependence of the potential energy barrier against antivortex entry is given by the function

$$
V_{i n}^{-}(N, v)=\mathcal{F}_{-}(N, v)-\mathcal{F}_{0}(N),
$$

so that $V_{i n}^{-}(N, b)=0$, while $V_{i n}^{-}(N, a)=\epsilon_{0}(2 h-2 N+1)$, which corresponds to the energy of transition from the state $N$ to the state $N-1$. On the other hand, if an antivortex moves out, starting from the inner radius $a$, the spatial dependence of the potential energy barrier against vortex exit is given by the function

$$
V_{\text {out }}^{-}(N, v)=\mathcal{F}_{-}(N+1, v)-\mathcal{F}_{0}(N),
$$

such that $V_{\text {out }}^{-}(N, a)=0$, while $V_{\text {out }}^{-}(N, b)=\epsilon_{0}(2 N+1-$ $2 h)$, which corresponds to the energy of transition from the state $N$ to the state $N+1$.

Note that $V_{\text {out }}^{-}(N, v)$ differs from $V_{\text {in }}^{-}(N+1, v)$ only by a constant: $V_{\text {out }}^{-}(N, v)-V_{\text {in }}^{-}(N+1, v)=\mathcal{F}_{0}(N+1)-$ $\mathcal{F}_{0}(N)=\epsilon_{0}(2 N+1-2 h)$. When this constant is zero, i.e. when $h=N+1 / 2$, the barriers $V_{\text {out }}^{-}(N-1, v)$ and $V_{i n}^{-}(N, v)$ become identical.

We conclude the discussion of barriers by pointing out that although the total energies $\mathcal{F}_{ \pm}(N, H, v)$ are quadratic in $N$ and $H$, the barrier functions are linear in these variables. For example, we have for a vortex entry at $b$ :

$$
\begin{aligned}
& V_{i n}^{+}(N, H, v)=\epsilon_{v}(v) \\
& +\epsilon_{0}\left\{\left[2 N+\frac{\ln (b / v)}{\ln (b / a)}\right] \frac{\ln (b / v)}{\ln (b / a)}-2 h \frac{b^{2}-v^{2}}{b^{2}-a^{2}}\right\} .
\end{aligned}
$$

\section{NARROW RINGS}

The energy (38) simplifies for narrow rings of width $W=b-a \ll a$, i.e., for

$$
\eta=W / a<<1
$$

We obtain in the linear approximation in $\eta$ :

$$
\begin{aligned}
\mathcal{F}_{ \pm} & =\epsilon_{v}(z)+\epsilon_{0}(N \pm z-h)^{2} \\
\epsilon_{v} & =\frac{\phi_{0}^{2}}{8 \pi^{2} \Lambda} \ln \left(\frac{2 a \eta}{\pi \xi} \sin \pi z\right), \quad z=\frac{b-v}{b-a} .
\end{aligned}
$$

Here, $0<z<1$; the energy and field scales are

$$
\epsilon_{0} \approx \frac{\phi_{0}^{2}}{8 \pi^{2} \Lambda} \eta, \quad H_{0} \approx \frac{\phi_{0}}{\pi a^{2}} .
$$

In particular, we have for the vortex-free state $(z=0)$ :

$$
\mathcal{F}_{0} \approx \frac{\phi_{0}^{2} \eta}{8 \pi^{2} \Lambda}(N-h)^{2}=\frac{\phi_{0}^{2} \eta}{8 \pi^{2} \Lambda}\left(N-\frac{\pi a^{2} H}{\phi_{0}}\right)^{2}
$$
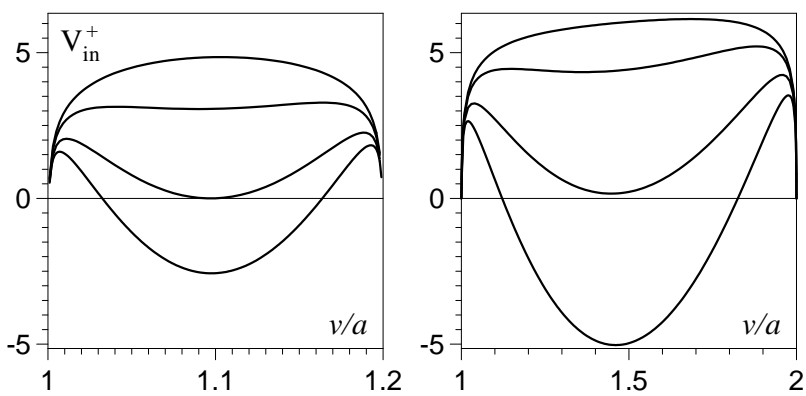

FIG. 3: The energy barriers $V_{i n}^{+}(N, H, v)$ for the vortex entry at the outer radius $b$ calculated numerically according to Eq. (44). The energy $V_{i n}^{+}$is given in units of $\phi^{2} / 8 \pi^{2} \Lambda$. The vortex position $v$ is normalized on the inner radius $a$. The left panel: $b / a=1.2$; the parameters from top to bottom are $h=N=$ $3,110,295$, and $450 ; h$ is normalized to $H_{0}$ of Eq. (39). The right panel: $b / a=2$; the parameters from top to bottom are $h=N=3,10,28$, and 50. Note: the normalization field $H_{0}$ for this set differs from the first.

an expression similar to that for thin cylinders 4.20

The potential barriers defined in Eqs. (44)-(47) are now easily evaluated. We focus here on $V_{i n}^{+}$for a vortex, which reads in linear approximation in $\eta$ :

$$
V_{i n}^{+} \approx \epsilon_{v}+\epsilon_{0} z(2 N-2 h+z) .
$$

One readily verifies that this potential reaches maximum at $z_{m}=1 / 2+\mathcal{O}(\eta)$, i.e., at $v_{m}=(b+a) / 2$.

To study the behavior of the potential $V_{i n}^{+}(N, H, v)$ near its maximum in increasing fields, one should go to higher order terms in the small $\eta$. To avoid cumbersome algebra, we use the fact that in the ground state $|h-N| \leq 1 / 2$ so that in large fields we can set $N \approx h$ and study the behavior of the function $V_{i n}^{+}(h, h, v)$. Of a particular interest is the curvature of the potential barrier at $v=v_{m}$. Numerical experimentation shows that in large fields, the maximum of $V_{i n}^{+}$is situated close to $v_{m}=(b+a) / 2$. Differentiating twice the function $V_{i n}^{+}(h, h, v)$ with respect to $v$ (this is easily done using Mathematica) and setting $v=v_{m}=a+W / 2$, we find that the curvature turns zero at

$$
H \approx H_{1}=\frac{\pi \phi_{0}}{4 W^{2}}
$$

For $H>H_{1}$, the potential barrier $V_{i n}^{+}\left(h, h, v_{m}\right)$ acquires a local minimum. Examples are shown in Fig. 3. A vortex at this minimum is in a metastable state provided $V_{i n}^{+}\left(h, h, v_{m}\right)>0$.

With increasing field, the depth of the minimum increases and at a field that may be called the low critical field, $H_{c 1}, V_{i n}^{+}\left(h, h, v_{m}\right)$ becomes equal to zero. This is the minimum field at which vortices can nucleate at the ring and stay there in stable equilibrium. We can estimate this field by setting $V_{i n}^{+}\left(h, h, v_{m}\right)=0$ in Eq. (48). For narrow rings we obtain:

$$
H_{c 1} \approx \frac{2 \phi_{0}}{\pi W^{2}} \ln \frac{2 W}{\pi \xi} .
$$


Clearly, with the further field increase, the barriers on both sides of the minimum are suppressed, while the points $v_{0}$ where $V_{i n}^{+}=0$ are pushed towards the annulus edges at $a$ and $b$. The critical field $H^{*}$ at which the "edge barrier" near $b$ disappears can be estimated by setting $V_{i n}^{+}\left(h, h, v_{0}\right)=0$ in Eq. (48) and considering the limit $v_{0} \approx b-\xi$. After straightforward algebra we obtain:

$$
H^{*} \approx \frac{\phi_{0} \ln 2}{\pi \xi b} \frac{\ln (b / a)}{2 \ln (b / a)-1+a^{2} / b^{2}} .
$$

For narrow rings, this reduces to

$$
H^{*} \approx \frac{\phi_{0} \ln 2}{2 \pi \xi W}
$$

whereas for rings with a small hole, $a \ll b$,

$$
H^{*} \approx \frac{\phi_{0} \ln 2}{2 \pi \xi b}\left[1+\frac{1}{2 \ln (b / a)}\right] .
$$

It is of interest to note that the field $H_{1}$ of Eq. (55) is temperature independent, $H_{c 1}$ of Eq. (56) is only weakly depends on $T$, whereas $H^{*}$ vanishes as $T \rightarrow T_{c}$ as $\sqrt{1-T / T_{c}}$. The last two are to be compared with the bulk $H_{c 1}$ and $H^{*}$, the bulk characteristics of the BeanLivingston barrier, both of which are linear in $1-T / T_{c}$ near $T_{c}$

\section{DISCUSSION}

The behavior of narrow rings is closely related to that of long strips. The field given in Eq. (55) was derived previously in a study of long strips of width $W$, where $H_{1}$ was called the vortex exclusion field ${ }^{21}$ According to this theory, when a superconducting strip is cooled through $T_{c}$ in an ambient magnetic field $H_{a}$, vortices should be excluded from the strip when $H_{a}$ is less than $H_{1}$, because the free-energy then has a global maximum at the strip center (at $v_{m}$ in our notation). On the other hand, when $H_{a}$ is greater than $H_{1}$, there is a local free-energy minimum at $v_{m}$, where the probability of finding a vortex is proportional to $\exp \left(-V_{\min }^{+} / k_{B} T\right)$. Near $T_{c}$, this probability is close to unity. However, as $T$ decreases, the characteristic energy scale, $\phi_{0}^{2} / 8 \pi^{2} \Lambda(T)$, increases rapidly. The vortices are then "frozen in" a potential well similar to those shown in Fig. 3 at a characteristic freeze-in temperature $T_{f}$, which for most superconductors is estimated as being very close to $T_{c}$. The value of $\Lambda\left(T_{f}\right)$ turns out larger than strip widths of the order of a few $\mu \mathrm{m}$, which justifies calculations done in the thin-film limit. The above arguments suggest that SQUIDs to be cooled and operated in the earth's magnetic field should be made entirely of narrow lines in order to avoid flux noise due to thermal agitation of vortices trapped in the lines. Experiments by Dantsker et al $\underline{22}^{22}$ are consistent with this conclusion.

The solutions presented here for currents and energies in thin mesoscopic superconducting rings are of importance for the physics of isolated rings (such as in Ref.
3), as well as for understanding the behavior of large ensembles of interacting rings $\frac{1.2}{.}$ The statistical mechanics of these systems requires knowledge of ring energy levels and probabilities of quantum or thermally activated transitions between the states of different energies. Our work should be useful for such statistical modelling. Despite the known shortcomings of the London approach, this is the only method that is of practical use for temperatures away from the critical temperature. A key advantage of the London equations is their linearity, which makes exact solutions for mesoscopic rings possible.

The electrostatic analogy we employed, may prove useful for various mesoscopic sample shapes. A number of these shapes (as squares, rectangles, or polygons) can be found in textbooks on applications of the theory of complex functions to the two-dimensional electrostatics, see, e.g., Ref. 16. An example of a thin-film disk is considered in the Appendix.

\section{Acknowledgments}

We are glad to thank John Kirtley for numerous discussions. In part this research is supported by Grant No. 2000011 from the United States-Israel Binational Science Foundation (BSF), Jerusalem, Israel. This manuscript has been authored in part by Iowa State University of Science and Technology under Contract No. W-7405Eng-82 with the U.S. Department of Energy.

\section{APPENDIX A: THIN FILM DISK}

It is worth stating upfront that the solution for a disk cannot be obtained by setting the inner radius $a=0$ in the solutions for a ring. The ring topology differs from that of a disk, and there is no continuous transition from the one to another. In fact, for a disk with no vortices the vorticity $N \equiv 0$. The state of a disk is determined by the continuous variable $H$, the applied field, and by the vortex position (or positions, if any).

Consider a thin-film disk of a radius $b \ll \Lambda$ with a vortex at an arbitrary position $\boldsymbol{v}$. To find the current distribution one has to solve Eq. (8) with the boundary condition $G(r=b)=$ const $=G_{b}$, or alternatively, Eqs. (9) for $G_{v}$ and (10) for $G_{H}$ under boundary conditions $G_{v}(b)=0$ and $G_{H}(b)=G_{b}$.

The part $G_{H}$ reads:

$$
G_{H}(r)=\frac{c H}{8 \pi \Lambda} r^{2}+G_{0} \ln \frac{r}{r_{0}},
$$

where the constants $G_{0}$ and $r_{0}$ are to be determined using the fact that the total current is $I=-G_{b}$. Evaluating $I$ with the help of London equation (15) one should take the phase change on circles $r<v$ as zero, whereas for $r>v$ as $-2 \pi$. Doing this algebra we obtain:

$$
G_{0}=-\frac{c \phi_{0}}{4 \pi^{2} \Lambda}, \quad r_{0}=v,
$$


and

$$
G_{H}(r)=\frac{c H}{8 \pi \Lambda} r^{2}-\frac{c \phi_{0}}{4 \pi^{2} \Lambda} \ln \frac{r}{v} .
$$

The problem of finding the part $G_{v}$ is equivalent to one for the electrostatic potential of a linear charge $c \phi_{0} / 8 \pi^{2} \Lambda$ at the point $\boldsymbol{v}$ parallel to the grounded metallic cylinder of a radius $b$. The solution is given, e.g., in Ref. 19:

$$
G_{v}=-\frac{c \phi_{0}}{4 \pi^{2} \Lambda} \ln \left(\frac{b}{v} \sqrt{\frac{r^{2}+v^{2}-2 r v \cos \varphi}{r^{2}+x^{2}-2 r x \cos \varphi}}\right),
$$

where $r, \varphi$ are cylindrical coordinates, the zero azimuth is taken as that of $\boldsymbol{v}$, and $x=b^{2} / v$. As discussed, the vortex self-energy $\epsilon_{v}=\phi_{0}\left|G_{v}(\varphi=0, r \rightarrow v)\right| / 2 c$ is logarithmically divergent, so that we set $r=v+\xi$ and $\varphi=0$ to obtain:

$$
\epsilon_{v}=\frac{\phi_{0}^{2}}{8 \pi^{2} \Lambda} \ln \frac{b^{2}-v^{2}}{b \xi} .
$$

The following is obtained in the same way as in the main text. The energy in zero field is

$$
E(0, v)=\epsilon_{v}(v)+\frac{\phi_{0}^{2}}{8 \pi^{2} \Lambda} \ln \frac{b}{v},
$$

and the magnetic moment is

$$
\mu=\frac{\phi_{0}\left(b^{2}-v^{2}\right)}{8 \pi \Lambda}-\frac{H b^{4}}{16 \Lambda} .
$$

The thermodynamic potential $\mathcal{F}=E(0, v)-\int_{0}^{H} \mu d H$ follows:

$$
\mathcal{F}=\frac{\phi_{0}^{2}}{8 \pi^{2} \Lambda}\left(\ln \frac{b^{2}-v^{2}}{b \xi}-h \frac{b^{2}-v^{2}}{b^{2}}-\frac{h^{2}}{4}\right),
$$

where $h=\pi b^{2} H / \phi_{0}$ (note that the field normalization here differs from the main text). In the vortex absence, $\mathcal{F}_{0}=\left(\phi_{0}^{2} / 32 \pi^{2} \Lambda\right) h^{2}$, so that the barrier for the vortex entry is

$$
V_{i n}^{+}=\frac{\phi_{0}^{2}}{8 \pi^{2} \Lambda}\left(\ln \frac{b^{2}-v^{2}}{b \xi}-h \frac{b^{2}-v^{2}}{b^{2}}\right) .
$$

It is now easy to find the field at which a local minimum first appears in the disk center $\left(d^{2} V_{i n}^{+} / d r^{2}=0\right.$ at $\left.v=0\right)$ :

$$
H_{1}=\frac{\phi_{0}}{\pi b^{2}}
$$

The field $H_{c 1}$ is determined by $V_{i n}^{+}(0)=0$ :

$$
H_{c 1}=\frac{\phi_{0}}{\pi b^{2}} \ln \frac{b}{\xi}
$$

the result given by Fetter 14

Thus for $H_{1}<H<H_{c 1}$, the vortex in the disk center is in metastable state, which becomes stable for $H>$ $H_{c 1}$. Finally, the barrier to the vortex entry disappears altogether when the point $v_{0}$ at which $V_{i n}^{+}=0$ moves to the edge, $v_{0}=b-\xi$ :

$$
H^{*}=\frac{\phi_{0} \ln 2}{2 \pi \xi b} .
$$

1 D. Davidovic̀, S. Kumar, D.H. Reich, J. Siegel, S.B. Field, R.C. Tiberio, R. Hey, and K. Ploog, Phys. Rev. Lett. 76, 815 (1996).

2 D. Davidovič, S. Kumar, D.H. Reich, J. Siegel, S.B. Field, R.C. Tiberio, R. Hey, and K. Ploog, Phys. Rev. B 55, 6518 (1997).

3 J.R. Kirtley, C.C. Tsuei, V.G. Kogan, J.R. Clem, H. Raffy, and Z.Z. Li, cond-mat/0302415

${ }^{4}$ M. Tinkham, Introduction to Superconductivity, McGrowHill, New York, 1996.

5 A. Bezryadin, A. Buzdin, B. Pannetier, Phys. Lett. A 195, 373 (1994).

6 J. Berger and J. Rubinstein, Phil. Trans. R. Soc. Lond. A 355, 1969 (1997).

7 J.J. Palacios, Phys. Rev. Lett. 84, 1796 (2000).

${ }^{8}$ F.M. Peters, V.A. Sweigert, B.J. Buelus, P.S. Deo, Physica C 332, 255 (2000).

${ }^{9}$ L.B. Ioffe, V.B. Geshkenbein, M.V. Feigel'man, A.L. Fauchère, G. Blatter, Nature 398, 679 (1999).

10 J.E. Mooij, T.P. Orlando, L. Levitov, Lin Tian, C.H. Van der Wal, and S. Lloyd, Science 285, 1036 (1999).
11 C. Van der Wal, A.C.J. ter Haar, F.K. Wilhelm, R.N. Schouten, C.J.P.M. Harmaks, T.P. Orlando, S. Lloyd, J.E. Mooij, Science 290, 773 (2000).

12 J. Pearl, Appl. Phys. Lett. bf 5, 65 (1964).

13 P.G. de Gennes, Superconductivity of Metals and Alloys, New York, Addison-Wesley, 1989.

14 A.L. Fetter, Phys. Rev. B 22, 1200 (1980).

15 V.G. Kogan, Phys. Rev. B 49, 15874 (1994).

16 P.M. Morse and H. Feshbach, Methods of theoretical physics, McGrow Hill, New York, 1953; part II, ch. 10.

17 Handbook of Mathematical Functions, edited by M. Abramowitz and A. Stegun, Natl. Bur. Stand. Appl. Math. Ser. No. 55 (U.S. GPO, Washington, D.C., 1965).

18 It is instructive to compare the vortex energy at the ring, Eq. (24) with an expression for a narrow straight strip: $\epsilon_{v}=\left(\phi_{0}^{2} / 8 \pi^{2} \Lambda\right) \ln [(2 W / \pi \xi) \sin (\pi v / W)]$, where $W \ll \Lambda$ is the strip width and $v$ is the vortex distance from one of the edges. ${ }^{15}$ One should mention that the general result for a vortex near the edge of a half-infinite thin film of Ref. 15 is erroneous; still, the discussion of energies and of the samples small on the scale of $\Lambda$ is correct. 
19 L.D. Landau and E.M. Lifshitz, Electrodynamics of continuous media, Pergamon, Oxford, New York, 1984.

20 A. Barone and G. Paterno, Physics and Applications of the Josephson Effect, Wiley, New York, 1982, p. 355.
21 J.R. Clem, unpublished.

22 E. Dantsker, S. Tanaka, and J. Clarke, Appl. Phys. Lett. 70, 2037 (1997). 